\title{
6-hydroxydopamine (6-OHDA) Oxidative Stress Assay for Observing Dopaminergic Neuron Loss in Caenorhabditis elegans \\ Sarah-Lena Offenburger ${ }^{\$, ~ *}$ and Anton Gartner
}

\begin{abstract}
Centre for Gene Regulation and Expression, School of Life Sciences, University of Dundee, Dundee, United Kingdom; ${ }^{\$}$ Current/Present address: EMBL-CRG Systems Biology Unit, Centre for Genomic Regulation (CRG), The Barcelona Institute for Science and Technology, Barcelona, Spain *For correspondence: sarah.offenburger@crg.eu
\end{abstract}

[Abstract] The nematode Caenorhabditis elegans is a powerful genetic model that can be used to investigate neuronal death. Research using $C$. elegans has been crucial to characterize cell death programmes that are conserved in mammals. Many neuronal signaling components, such as those mediating dopaminergic neurotransmission, are preserved as well. Dopaminergic neurons are progressively lost in Parkinson's disease and an important risk factor to develop this disease appears to be oxidative stress, the increased occurrence of highly reactive oxygen species. Oxidative stressinduced dopaminergic neurodegeneration is mimicked in animal models by treatment with 6hydroxydopamine (6-OHDA), a dopamine analog, which is specifically taken up into dopaminergic neurons. After exposing $C$. elegans to 6-OHDA, the loss of fluorescently labeled dopaminergic neurons can be easily monitored. An organisms' sensitivity to oxidative stress is thought to be influenced by basal levels of intrinsic oxidative stress and the ability to counteract oxidative stress and oxidative stressinduced damage. The $C$. elegans ' 6 -OHDA model' led to the discovery of novel genes that are required to protect dopaminergic neurons and it has helped to determine the effects of conserved cell death and cell engulfment pathways in dopaminergic neurodegeneration. Here, we describe a simple protocol that allows for the easy detection of dopaminergic neuron loss after 6-OHDA treatment in $C$.elegans.

Keywords: C. elegans, Caenorhabditis elegans, 6-OHDA, 6-hydroxydopamine, Oxidative stress assay, Intoxication, Dopaminergic neurodegeneration

[Background] The gradual loss of dopaminergic neurons can be recapitulated in animal models following exposure to the oxidative stress-inducing drug 6-hydroxydopamine (6-OHDA) (for review Schober, 2004). In contrast to other neurodegenerative drugs such as MPTP (1-methyl-4-phenyl1,2,3,6-tetrahydropyridine), 6-OHDA is safer to handle as it does not pass the blood-brain-barrier. 6OHDA is a hydroxylated dopamine analog, which is specifically taken up into dopaminergic neurons by the dopamine transporter and blocks complex I of the respiratory chain (Schober, 2004). The resulting formation of reactive oxygen species is thought to trigger 6-OHDA-induced neurodegeneration (Schober, 2004).

6-OHDA exposure of the nematode Caenorhabditis elegans leads to the selective loss of dopaminergic neurons (Nass et al., 2002) and components of dopaminergic neurotransmission are highly conserved compared to mammals (for review Nass et al., 2001). C. elegans hermaphrodites 
possess eight dopaminergic neurons: four CEP (cephalic sensilla) and two ADE (anterior deirids) dopaminergic neurons in the head, and two PDE (posterior deirids) dopaminergic neurons in the midbody (Sulston et al., 1975). These neurons can be specifically labeled by expression of a fluorescent protein driven by the promoter of the dat-1 dopamine transporter (Nass et al., 2002). The C. elegans 6OHDA model can be used to understand how dopaminergic neurons maintain their integrity when subjected to oxidative stress.

The first published 6-OHDA intoxication protocol for $C$. elegans used high concentrations of 6-OHDA elicit dopaminergic neurodegeneration in wild-type animals (Nass et al., 2002). Mutation of the dopamine transporter dat-1, which is required for neuronal 6-OHDA uptake, was shown to confer 6-OHDA resistance (Nass et al., 2002). After 6-OHDA exposure during larval stages (L3 and L4), dopaminergic neurodegeneration was scored in a low-throughput manner by mounting adult animals on cover slide (Nass et al., 2002; Tucci et al., 2011). We adapted the protocol to screen for mutants that are hypersensitive to 6-OHDA exposure by using lower 6-OHDA concentrations that do not elicit neurodegeneration in wild-type animals. We expose synchronized L1 stage larvae in 96-well plates and score adults directly on agar plates, allowing for high-throughput screening. This approach led to the characterization of the tetraspanin gene $t s p-17$, the neuroligin-like gene glit-1 and the transthyretinrelated gene ttr-33, all of which protect $C$. elegans dopaminergic neurons from 6-OHDA-induced neurodegeneration (Masoudi et al., 2014; Offenburger et al., 2018a and 2018b). The C. elegans 6OHDA assay was further used to describe the roles of known stress response and cell death pathways in oxidative stress-induced dopaminergic neurodegeneration (Nass et al., 2002; Tóth et al., 2007; Offenburger et al., 2018a and 2018b).

The protocol can also be used for acute liquid exposure to other soluble compounds such as the oxidative stress-inducing drug paraquat (Offenburger et al., 2018a and 2018b). The procedures we describe here are generally useful to test if compounds influence dopaminergic neuron death.

\section{Materials and Reagents}

1. Latex gloves

2. Lab coat

3. Platinum wire (e.g., CVS10 replacement platinum wire, $50 \mathrm{~cm}$ length $\times 0.2 \mathrm{~mm}$ diameter, SigmaAldrich, catalog number: EP1330)

4. High recovery filter pipette tips (e.g., Corning, Axygen ${ }^{\circledR}$ Maxymum Recovery ${ }^{\circledR}$, catalog number: T-200-C-L-R-S)

5. Bench surface protector sheets (GE Healthcare, Whatman ${ }^{\circledR}$, catalog number: 2300-916)

6. Disposable spatulas (e.g., Disposable smartSpatula, LevGo, catalog number: 17251)

7. $1.5 \mathrm{ml}$ screw cap tubes (sterile, graduated, conical, e.g., STARLAB, catalog number: E14152231)

8. 96-well plates (Tissue culture plates, round bottom, clear, sterile, TPP Techno Plastic Products, catalog number: 92097) 
9. Only if filtering of $\mathrm{L} 1$ larval stages required: Nylon net filter, hydrophilic, $5 \mu \mathrm{m}$ pore size (e.g., Merck, catalog number: NY0509050 with 90 mm diameter, or catalog number: NY0502500 with $25 \mathrm{~mm}$ diameter)

10. $10 \mathrm{~cm}$ plastic Petri dishes

11. Wet paper towel

12. E. coli OP50 [Caenorhabditis Genetics Centre (CGC), University of Minnesota, Dept of GCD, 6160 Jackson Hall, 321 Church Street S.E. Minneapolis, MN 55455, https://cgc.umn.edu/]

13. Experimental control strains available at the C. elegans Genetics Centre (CGC), University of Minnesota, https://cgc.umn.edu/):

a. TG2435 wild type-backcrossed BY200 derivate, vtls1[pdat-1::gfp; rol-6] V (while pdat-1::gfp is always expressed, the penetrance of the roller phenotype is very low, only rarely detectable)

b. TG2400 dat-1(ok157) III; vt/s1V

c. TG4100 vtls1 V; glit-1(gt1981) X

d. TG2436 vtls1 V; tsp-17(tm4995) $\mathrm{X}$

e. TG4103 ttr-33(gt1983) V; vt/s1 V

Note: We advise researchers to backcross strains to their wild-type strain for at least 4 times.

14. M9 buffer (He, 2011)

15. Nematode growth medium (NGM) agar ( $\mathrm{He}, 2011)$

16. LB (Luria-Bertani) liquid medium (see Cold Spring Harbor Protocols, 2006)

17. 6-hydroxydopamine (6-OHDA) hydrochloride (Sigma-Aldrich, catalog number: H4381) (keep at $-20{ }^{\circ} \mathrm{C}$ in the dark, prepare stock solution freshly)

18. L-ascorbic acid (Sigma-Aldrich, catalog number: A5960, $\geq 90 \%$ ) (store at room temperature in the dark, wrap aliquots with aluminum foil)

19. $200 \mathrm{mM}$ ascorbic acid solution (freshly prepared, see Recipes)

20. 10 mM 6-OHDA solution (freshly prepared, see Recipes)

\section{Equipment}

1. Pipettes

2. Metal inoculation loop

3. Precision scales

4. Temperature-controlled shaker (e.g., Eppendorf, model: ThermoMixer ${ }^{\circledR} \mathrm{R}$ )

5. Temperature-controlled incubator $\left(20^{\circ} \mathrm{C}\right)$

6. Fume hood

7. Vortex Mixer (e.g., Scientific Industries, model: Vortex-Genie 2)

8. Ethanol burner (e.g., DWK Life Sciences, Wheaton ${ }^{\mathrm{TM}}$ Alcohol Burner, catalog number: 237070)

9. Stereomicroscope with fluorescence (e.g., Leica) 


\section{Software}

1. R studio (version 1.0.44)

\section{Procedure}

A. Preparation

1. Prepare NGM plates and dry overnight at $37^{\circ} \mathrm{C}$. Store unseeded plates at $4{ }^{\circ} \mathrm{C}$.

2. Transfer the plates to room temperature before covering them with ca. $1 \mathrm{ml}$ of OP50 E. coli. We prepare E. coli cultures by inoculating a single colony of OP50 in $500 \mathrm{ml} \mathrm{LB}$ medium and overnight incubation at $37^{\circ} \mathrm{C}$.

3. Dry these seeded plates overnight and store them at $20^{\circ} \mathrm{C}$.

4. Maintain C. elegans at standard conditions at $20^{\circ} \mathrm{C}$ (Brenner, 1974) on $10 \mathrm{~cm}$ plastic Petri dishes containing nematode growth medium (NGM) agar and OP50 bacteria food.

Note: For the 6-OHDA experiments, the N2-derived BY200 C. elegans strain can be used as a wild-type control, dat-1 as a 6-OHDA-resistant strain (Nass et al., 2002) and the mutant strains tsp-17 (Masoudi et al., 2014), glit-1 (Offenburger et al., 2018b) or ttr-33 (Offenburger et al., 2018a) as 6-OHDA-sensitive mutants.

B. 6-OHDA assay (Figure 1)

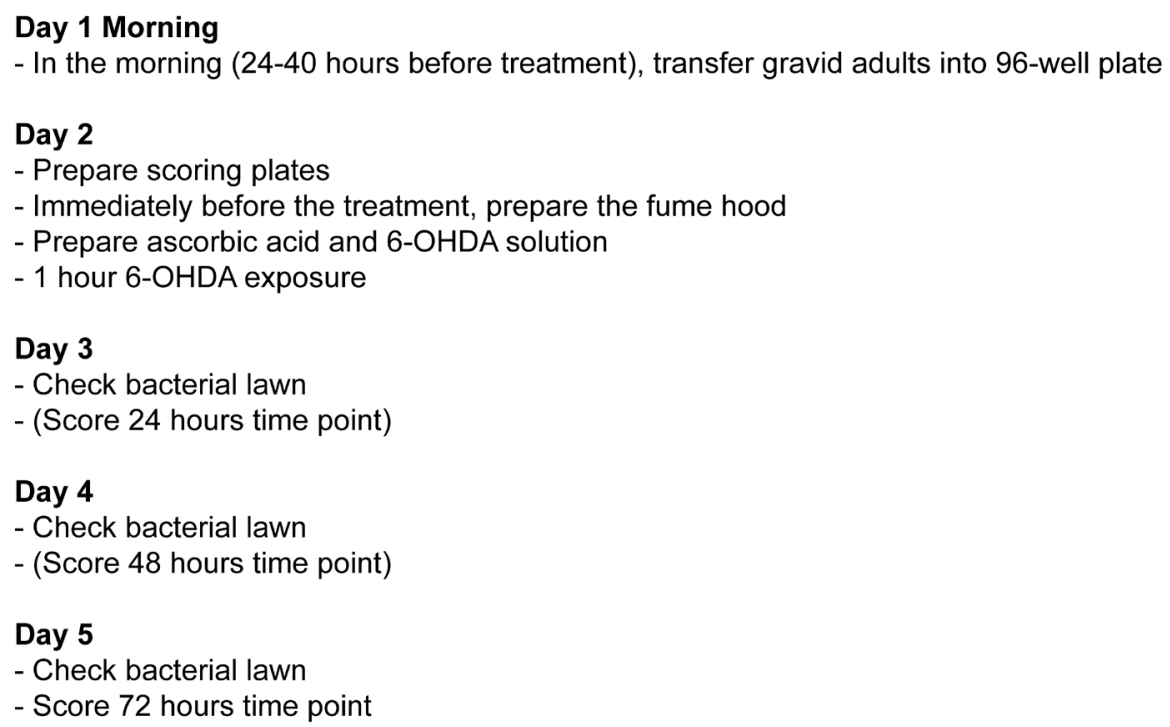

Figure 1. Overview of daily schedule of 6-OHDA assay. Please refer to text for details.

1. One day before the 6-OHDA assay, pick gravid $C$. elegans (in adult stage and full of eggs) into a 96-well plate containing $70 \mu \mathrm{l}$ M buffer per well. Only $30 \mu \mathrm{l}$ of these $70 \mu \mathrm{l}$ will be used for the 6-OHDA treatment, but as a substantial amount of the liquid will evaporate, it is necessary to prepare a larger volume. It is best practice to select healthy day 1 or day 2 adults for all 
conditions and replicates.

Note: We recommend avoiding wells on the edge of the plate (rows $A$ and $H$, and columns 1 and 12, respectively), as liquids in the outermost wells show increased evaporation.

2. Transfer 10 adults into each well using a platinum wire, minimizing as little bacteria carryover as possible, as bacterial growth in the 96-well plates would compromise the synchronization of C. elegans L1 stage larvae.

Note: If necessary, first transfer worms to an empty NGM plate for a few seconds and then move clean animals that have crawled away from the bacteria to the 96-well plate.

3. Prepare a technical duplicate for each experiment (i.e., two wells per condition) and perform at least two biological replicates on different days. We encourage randomizing the sequence of the analyzed strains (Figure 2).

A
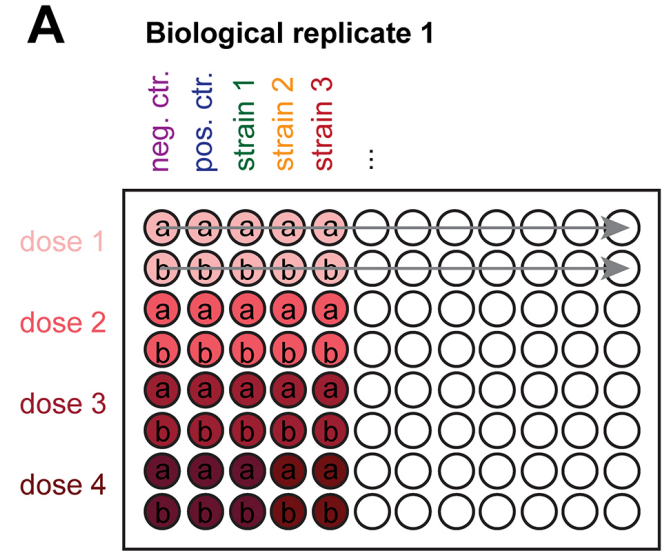

B

Biological replicate 2

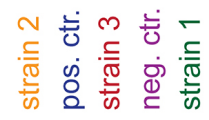

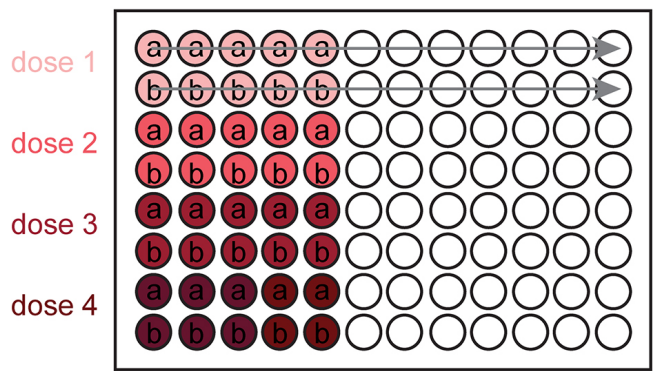

Figure 2. Schematic representation of oxidative stress assay design. C. elegans are exposed in liquid in 96-well plates. The assay is run in technical duplicate (rows a $+b$ per dose) including negative and positive controls. We encourage randomizing the position of control strains between the biological replicates $(A+B)$. The biological replicates must be performed on separate days. The red color gradient indicates the increasing dose of the drug. We commonly used doses of $0.75 \mathrm{mM}, 10 \mathrm{mM}, 25 \mathrm{mM}$ and $50 \mathrm{mM}$ 6-OHDA. We recommend avoiding wells on the edge of the plate (row $A$ and $H$ and column 1 and 12), as liquids in the outermost wells show increased evaporation. We maintain the same pipetting sequence (indicated with a grey arrow) when adding compounds to ensure equal exposure of all tested strains.

4. Incubate the 96 -well plates in a temperature-controlled shaker at $500 \mathrm{rpm}$ at $20{ }^{\circ} \mathrm{C}$ for $24-40 \mathrm{~h}$. As food is absent in the wells, the eggs laid by these adults will hatch yet remain in the L1 larval stage, thus providing a developmentally synchronous culture. We use $30 \mathrm{~h}$ of incubation time for all our experiments and encourage standardizing this time interval as starvation renders the animals more resistant to 6-OHDA treatment (González-Hunt et al., 2014; Offenburger et al., 2018b). After $30 \mathrm{~h}$, several hundred L1s will accumulate in the wells, of which only around 200 
will be used in the assay and only 50-100 randomly selected animals will be scored after treatment.

5. On the day of 6-OHDA treatment, first prepare the recovery plates. Label NGM agar-containing $6 \mathrm{~cm}$ plates in the back with the respective letter/number code from the 96-well plate and streak a line of OP50 E. coli on the NGM using a metal inoculation loop (Figure 3A).

Notes:

a. Other items can be used to streak the line of OP50. However, it is important not to damage the agar surface as otherwise animals might bury into the agar and will thus be lost for scoring.

b. To ensure the food does not run out when analyzing a large number of animals, it is possible to streak concentrated bacteria (OP50 E. coli centrifuged for $5 \mathrm{~min}$ at $>15,000 \times \mathrm{g}$ and resuspended in 1/10 volume of LB medium).

6. Immediately before treating the animals, prepare a $200 \mathrm{mM}$ ascorbic acid stock solution and a $5 x$ stock solution of 6-OHDA in MilliQ water in screw cap tubes. $10 \mu$ ascorbic acid stock solution and $10 \mu \mathrm{l} 6-\mathrm{OHDA}$ stock solution are required per well in a total volume of $50 \mu \mathrm{l}$. The 1:5 dilution results in a final concentration of $40 \mathrm{mM}$ of ascorbic acid and 1x 6-OHDA (we commonly used, $0.75,10,25$ or $50 \mathrm{mM} 6-$ OHDA in our experiments). Ascorbic acid is an antioxidant, which prevents auto-oxidation of 6-OHDA.

Note: When handling 6-OHDA, wear double gloves and a lab coat, use disposable filter tips and work under the fume hood. Use a disposable bench cover in the fume hood and put a wet paper towel in the area in which 6-OHDA is handled such that spilled 6-OHDA is oxidized immediately.

7. Weigh ascorbic acid and 6-OHDA in the screw cap tubes using disposable spatulas and precision scales in the fume hood. To minimize handling time, weigh an approximate, slightly higher amount of compound and adapt the total volume accordingly.

8. Separate 6-OHDA waste materials and dispose of properly. Try to work with the same batch of $6-\mathrm{OHDA}$ as efficacies can vary between production lots. Vortex both the ascorbic acid solution and the 6-OHDA solution thoroughly for approximately $1 \mathrm{~min}$ until flakes are dissolved and the solutions appear homogenous.

Note: The screw caps help to ensure that that the tubes stay closed during mixing. If working with several different concentrations or with small concentrations, prepare dilution series from the same stock solution.

9. Aliquot $30 \mu \mathrm{l}$ of worms in M9 buffer (corresponding to over 200 animals) into new wells using non-adhesive filter tips to minimize animal loss.

Note: A precise multi-channel pipette can be used to transfer animals. Technical replicates are not intermixed at this stage. If animal density varies greatly between the wells, it can be adjusted by diluting the worms in M9 buffer to produce a total volume of $30 \mu \mathrm{l}$.

10. Check with a stereomicroscope if a sufficiently high number of animals (at least 50 animals per well) have been transferred before adding the compounds.

11. Then add $10 \mu \mathrm{l}$ ascorbic acid solution and knock 96 -well plate on the bench or centrifuge the 
plate shortly to make sure the contents are mixed properly before adding 6-OHDA.

Note: No drops of ascorbic acid solution should remain on the wall of the well. Shake the plate shortly to ensure equal distribution of the ascorbic acid in the wells. To prevent auto-oxidation of 6-OHDA, the ascorbic acid must be added first. A precise multi-channel pipette can be used to dispense reagents.

12. Add $10 \mu \mathrm{l} 5 \times 6-\mathrm{OHDA}$ solution and again ensure the solutions are properly mixed. Shake the multiwell plates for $1 \mathrm{~h}$ at $500 \mathrm{rpm}$ at $20^{\circ} \mathrm{C}$.

13. Stop the incubation by adding $150 \mu \mathrm{M} 9$ buffer. This buffer will oxidize the $6-\mathrm{OHDA}$ and the solution will turn pink to dark red. The intensity of the color after addition of the buffer is a good indicator of the 6-OHDA concentration used.

14. Pipet the total of $200 \mu \mathrm{l}$ of worms into oxidized 6-OHDA solution on the NGM plates opposite the bacterial stripe, directly on top of the plate label (Figure 3B).

15. Open the lids and let the plates dry under the fume hood. When the 6-OHDA solution has soaked into the NGM, remove the adult animals and eggs with a platinum wire such that only animals that were exposed at L1 stage remain on the plate (Figure 3C). Unless working with mutant strains that exhibit a developmental defect, there are usually no eggs or very few eggs present as all of progeny will have hatched in the $24-40 \mathrm{~h}$ incubation time before the assay. The L1 larvae remaining on the scoring plates will migrate towards the bacterial streak, which provides the food source (Figure 3D).
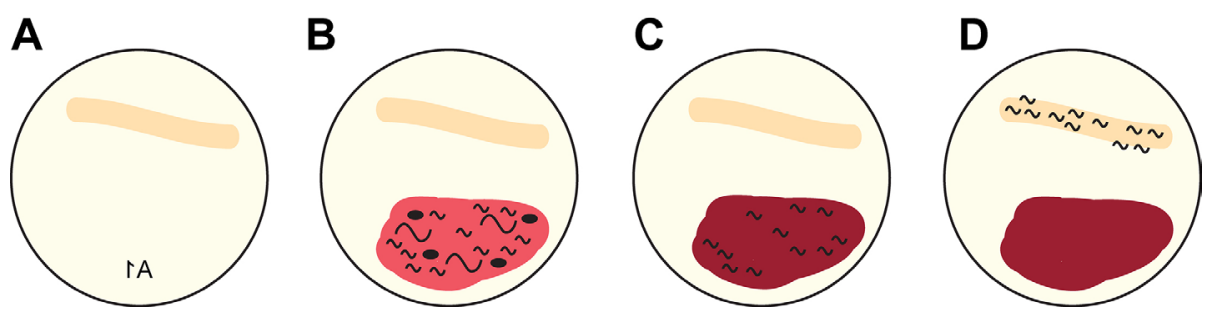

Figure 3. Layout of recovery plates. A. $6 \mathrm{~cm}$ NGM Petri dish labeled in the back with the well number (e.g., 1A) and inoculated with a stripe of OP50 E. coli (orange streak). B. The mixture of adult and L1 stage animals and unhatched eggs in oxidized 6-OHDA (red liquid) is added to the plate on the side opposite to the bacterial streak. C. Adult animals and unhatched eggs are removed with a platinum wire. D. The remaining L1 stage larvae will migrate towards the bacterial streak, allowing for convenient scoring.

16. Check the plates daily to make sure the bacterial food does not run out. In case there is little food left, add a drop (ca. 50-100 $\mu$ l) of concentrated E. coli to prevent animals from starvation. Dopaminergic neurodegeneration can be observed 24,48 or $72 \mathrm{~h}$ after exposure. The experimenter should be blinded to the genotype. C. elegans dopaminergic head neurons (Figure 4A) show differential sensitivity to 6-OHDA, with CEP neurons being more sensitive than ADE neurons (Nass et al., 2002; Tucci et al., 2011). PDE dopaminergic neurons in the midbody only appear after the L1 larval stage and are not scored in our experiments. Score at least 50 animals 
per replicate plate, resulting in a total number of at least 100 scored animals for each condition. Animals were selected randomly for scoring. We use four categories for scoring: (1) CEP + ADE neurons intact, (2) CEP neurons partially compromised and ADE neurons intact, (3) only ADE neurons remaining, and (4) no CEP or ADE neurons left (legend in Figure 4B, Table 1). This assay can be used for genetic screens to look for hypersensitive mutants (Figure 4B), to analyze epistasis between different mutations at differing concentrations of 6-OHDA (Figure 4C), or to analyze animals upon exposure to other soluble compounds such as paraquat (Figure 4D).

A

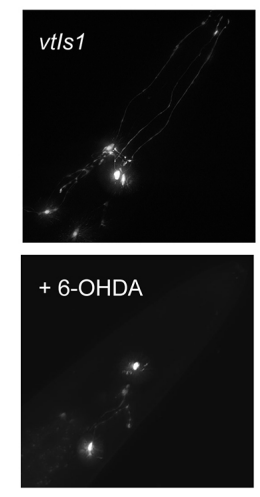

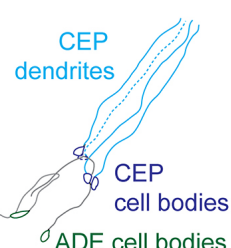

ADE cell bodies

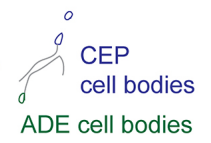

B

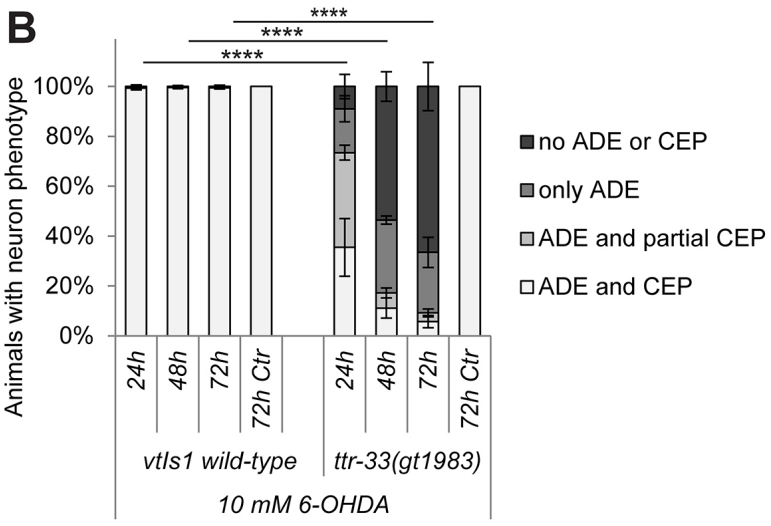

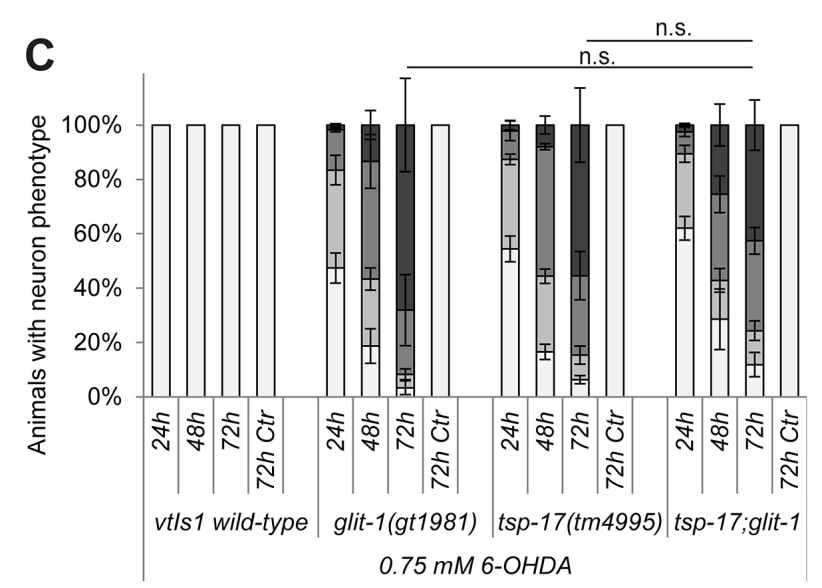

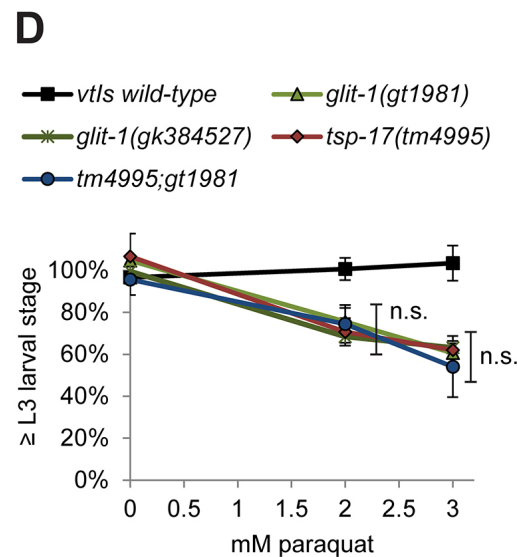

Figure 4. Representative readouts from oxidative stress assay. Panels $A$ and $B$ are adapted from Offenburger et al. (2018a) and panel C and D are adapted from Offenburger et al. (2018b). A. Fluorescently labeled $C$. elegans dopaminergic head neurons in the wild-type strain carrying the vt/s1 transgene (left) and schematic labeling (right) without (top) and with exposure to 50 mM 6-OHDA ('+6-OHDA', bottom) $72 \mathrm{~h}$ after treatment. ADE neurons are located posterior to CEP neurons. B. Remaining dopaminergic head neurons 24,48 and $72 \mathrm{~h}$ after exposure to 10 mM 6-OHDA and $72 \mathrm{~h}$ after control treatment with ascorbic acid only ('72h Ctr') in BY200 wildtype animals and in the isolated hypersensitive mutant ttr-33(gt1983). Error bars = SEM of 3 biological replicates, each with 60-120 animals per strain. Total number of animals per condition $\mathrm{n}=30$ for the ' $72 \mathrm{~h} \mathrm{Ctr'}$ and $\mathrm{n}=220-340$ for all the other conditions ( ${ }^{* * * *} P<0.0001$; G-Test comparing wild-type and mutant data of the same time point). C. Dopaminergic head neurons in wild-type and glit-1 and tsp-17 single and double mutants 24,48 and $72 \mathrm{~h}$ after treatment with 
$0.75 \mathrm{mM} 6-\mathrm{OHDA}$ and $72 \mathrm{~h}$ after control treatment with ascorbic acid only (' $72 \mathrm{~h}$ Ctr'). Error bars $=$ SEM of 2-3 biological replicates, each with 60-115 animals per strain. Total number of animals per condition $\mathrm{n}=60$ for the ' $72 \mathrm{~h}$ Ctr' condition and $\mathrm{n}=180-350$ for all the other conditions (n.s. $P>0.05$; G-Test). D. Percentage of animals that developed to $L 3$ stage $24 \mathrm{~h}$ after $1 \mathrm{~h}$ incubation with $50 \mathrm{mM}$ paraquat. Error bars = SEM of 3 biological replicates, each with 85-380 animals per strain and concentration. Total number of animals per condition $\mathrm{n}=340-830$ (n.s. $P>0.05$; twotailed $t$-test comparing the tsp-17(tm4995) and glit-1(gt1981) single mutants to the tsp17(tm4995);glit-1(gt1981) double mutant at $2 \mathrm{mM}$ and $3 \mathrm{mM}$ paraquat).

Table 1. Example scoring for strains a and b. Number of animals in each category (sum of technical duplicate).

\begin{tabular}{|l|l|l|l|l|}
\hline & ADE + CEP (1) & ADE + partial CEP (2) & Only ADE (3) & No neurons (4) \\
\hline Strain a & 12 & 2 & 18 & 86 \\
\hline Strain b & 20 & 5 & 28 & 54 \\
\hline
\end{tabular}

\section{Data analysis}

We visualized scoring results with the ' $100 \%$ Stacked Colum' chart in Microsoft Excel. We added up numbers scored in technical duplicates and excluded data points for which the standard deviation between the technical duplicates was higher than $20 \%$ in any scoring category.

To determine statistical significance, we performed the $G$-Test with the 'DescTools' package of $R$ Studio (version 1.0.44). The package is loaded with 'library(DescTools)'. To compare two strains a and $b$ with the respective number of neurons in the neuronal scoring category 1-4, the matrix 'mat' was defined by mat $=\operatorname{cbind}(c(1 a, 2 a, 3 a, 4 a), c(1 b, 2 b, 3 b, 4 b))$. A G-Test on this matrix was performed with 'GTest(mat)'. The result is given as a $P$-value.

Code for example above:

$>$ library (DescTools)

$>$ mat $=$ cbind $(\mathrm{c}(12,2,18,86), \mathrm{c}(20,5,28,54))$

$>$ GTest (mat)

Output for example above:

Log likelihood ratio (G-test) test of independence without correction

data: mat

$G=12.382, x$-squared $d f=3, p$-value $=0.006182$ 


\section{$\underline{\text { Notes }}$}

1. To compare results between strains, it is best practice to test them always in parallel as there will be variations between the assays.

2. To test drug response in $C$. elegans of different life cycle stages, expose a mixed population of animals to $6-\mathrm{OHDA}$ and transfer $C$. elegans of distinct stages to separate scoring plates using a platinum wire.

3. If working with a strain that exhibits a swimming defect (for example unc mutants), synchronize L1 stage animals without starvation by washing off a plate of mixed stage animals and passing them through a $5 \mu \mathrm{m}$ nylon net filter which will retain animals of all other stages except for L1.

4. Aim to use the same lot of 6-OHDA for all experiments as efficiencies between batches can vary. Also keep in mind that 6-OHDA might oxidize over time after opening, so older batches might become less efficient over time.

5. The ascorbic acid solution and the 6-OHDA solution must be prepared freshly immediately before treating the animals to avoid oxidization of the compounds.

6. Prepare at least $100 \mu \mathrm{l}$ of each ascorbic acid solution and 6-OHDA solution, as otherwise the amounts will be too small to weigh them out accurately.

7. The solutions should appear transparent until addition of M9 buffer. Pink solutions indicate oxidation of 6-OHDA, which does not cause neurodegeneration.

8. A higher number of animals can be treated in screw cap tubes and a higher total volume, e.g., in preparation for a qRT-PCR. Synchronized L1 for large approaches can be obtained by starvation of a big plate (keeping the starvation time as short as possible) or by filtering a big, mixed population of animals with a $5 \mu \mathrm{m}$ nylon net filter.

9. If the protocol is used for the paraquat assay, pipet a limited number of animals (ca. 100) on the scoring plates. Count the total number of animals transferred to express the number of animals reaching L3 stage as a percentage.

\section{$\underline{\text { Recipes }}$}

1. $200 \mathrm{mM}$ ascorbic acid solution (100 $\mu \mathrm{l})$

Weigh $3.52 \mathrm{mg}$ ascorbic acid (MW = $176.12 \mathrm{~g} / \mathrm{mol}$ )

Vortex solution thoroughly for approximately 1 min until flakes are dissolved and the solution appears as homogeneous

2. $10 \mathrm{mM} 6-\mathrm{OHDA}$ solution $(100 \mu \mathrm{l})$

Weigh $1.03 \mathrm{mg}$ 6-OHDA (MW = $205.64 \mathrm{~g} / \mathrm{mol})$

Vortex solution thoroughly for approximately $1 \mathrm{~min}$ until it appears as homogeneous. 


\section{Acknowledgments}

This protocol was adapted from previous work (Nass et al., 2002; Masoudi et al., 2014) and we thank Neda Masoudi for advice. This work was funded by a Wellcome Trust Programme grant to AG (0909444/Z/09/Z, https://wellcome.ac.uk/funding) and a Parkinson's UK grant (G0912, https://www.parkinsons.org.uk/research/research-grants), together with infrastructure funding from a Wellcome Trust Strategic award (097045/B/11/Z). We acknowledge the Dundee Imaging Facility, which is supported by the Wellcome Trust Technology Platform award (097945/B/11/Z) and the MRC Next Generation Optical Microscopy award (MR/K015869/1). SLO was supported by a Ph.D. fellowship from the Molecular and Cellular Biology programme funded by the Wellcome Trust and by ISSF funding from the Wellcome Trust. Some strains were provided by the CGC, which is funded by NIH Office of Research Infrastructure Programs (P40 OD010440).

\section{Competing interests}

The authors declare that no competing interests exist.

\section{References}

1. Brenner, S. (1974). The genetics of Caenorhabditis elegans. Genetics 77(1): 71-94.

2. González-Hunt, C. P., Leung, M. C., Bodhicharla, R. K., McKeever, M. G., Arrant, A. E., Margillo, K. M., Ryde, I. T., Cyr, D. D., Kosmaczewski, S. G., Hammarlund, M. and Meyer, J. N. (2014). Exposure to mitochondrial genotoxins and dopaminergic neurodegeneration in Caenorhabditis elegans. PLoS One 9(12): e114459.

3. He, F. (2011). Common worm media and buffers. Bio-protocol/Bio101 1: e55.

4. LB (Luria-Bertani) liquid medium. (2006). Cold Spring Harb Protoc pdb.rec8141. doi:10.1101/pdb.rec8141.

5. Masoudi, N., Ibanez-Cruceyra, P., Offenburger, S. L., Holmes, A. and Gartner, A. (2014). Tetraspanin (TSP-17) protects dopaminergic neurons against 6-OHDA-induced neurodegeneration in C. elegans. PLoS Genet 10(12): e1004767.

6. Nass, R., Hall, D. H., Miller, D. M., 3rd and Blakely, R. D. (2002). Neurotoxin-induced degeneration of dopamine neurons in Caenorhabditis elegans. Proc Natl Acad Sci U S A 99(5): 3264-3269.

7. Nass, R., Miller, D. M. and Blakely, R. D. (2001). C. elegans: a novel pharmacogenetic model to study Parkinson's disease. Parkinsonism Relat Disord 7(3): 185-191.

8. Offenburger, S. L., Ho, X. Y., Tachie-Menson, T., Coakley, S., Hilliard, M. A. and Gartner, A. (2018a). 6-OHDA-induced dopaminergic neurodegeneration in Caenorhabditis elegans is promoted by the engulfment pathway and inhibited by the transthyretin-related protein TTR-33. PLoS Genet 14(1): e1007125. 
9. Offenburger, S. L., Jongsma, E. and Gartner, A. (2018b). Mutations in Caenorhabditis elegans neuroligin-like glit-1, the apoptosis pathway and the calcium chaperone crt-1 increase dopaminergic neurodegeneration after 6-OHDA treatment. PLoS Genet 14(1): e1007106.

10. Schober, A. (2004). Classic toxin-induced animal models of Parkinson's disease: 6-OHDA and MPTP. Cell Tissue Res 318(1): 215-224.

11. Sulston, J., Dew, M. and Brenner, S. (1975). Dopaminergic neurons in the nematode Caenorhabditis elegans. J Comp Neurol 163(2): 215-226.

12. Tóth, M. L., Simon, P., Kovacs, A. L. and Vellai, T. (2007). Influence of autophagy genes on ionchannel-dependent neuronal degeneration in Caenorhabditis elegans. J Cell Sci 120(Pt 6): 1134-1141.

13. Tucci, M. L., Harrington, A. J., Caldwell, G. A. and Caldwell, K. A. (2011). Modeling dopamine neuron degeneration in Caenorhabditis elegans. In: Manfredi, G. and Kawamata H. (Eds.). Methods in Molecular Biology. Humana Press, 129-148. 